\title{
Biomarkers and Surrogate Outcomes in Neurodegenerative Disease: Lessons from Multiple Sclerosis
}

\author{
David H. Miller \\ Multiple Sclerosis NMR Research Unit, Department of Neuroinflammation, Institute of Neurology, London WC1N 3BG, \\ United Kingdom
}

Summary: Multiple sclerosis (MS) is a chronic disease of the CNS that most commonly affects young adults. It is usually characterized in the early years by acute relapses followed by partial or complete remission; in later years progressive and irreversible disability develops. Because of the protracted and unpredictable clinical course, biological surrogate markers are much needed to make clinical trials of potential disease-modifying treatments more efficient. Magnetic resonance (MR) outcome measures are now widely used to monitor treatment outcome in MS trials. Areas of multifocal inflammation are detected with a high sensitivity as new areas of gadolinium enhancement and T2 abnormality, and these may be considered as surrogate markers for clinical relapses. However, progressive disability is not clearly related to inflammatory lesions but rather to a progressive and diffuse process with increasing neuroaxonal loss. MR surrogate measures for neuroaxonal loss include atrophy (tissue loss in brain and spinal cord), $\mathrm{N}$-acetyl aspartate, and $\mathrm{T} 1$ hypointense lesions. Diffuse abnormality in normal appearing brain tissue may also be monitored using magnetization transfer ratio and other quantitative MR measures. For treatment trials of new agents aimed at preventing disability, measures of neuroaxonal damage should be acquired, especially atrophy, which occurs at all stages of MS and which can be quantified in a sensitive and reproducible manner. Because the MR surrogates for neuroaxonal loss are not yet validated as predicting future disability, definitive trials should continue to monitor an appropriate disability endpoint. Key Words: Multiple sclerosis, inflammation, neuroaxonal loss, magnetic resonance markers, treatment trials.

\section{THE CLINICAL COURSE AND PATHOLOGICAL BASIS OF MULTIPLE SCLEROSIS}

Multiple sclerosis (MS) is a chronic disorder of the CNS. It is the most common cause of neurological disability in young adults in Northern Europe, North America, and similar temperate latitudes in Australasia. It is conventionally regarded as an immune-mediated inflammatory disorder. The classic neuropathological hallmark is the presence of multifocal regions of inflammatory demyelination disseminated in space and time throughout the CNS. Such lesions develop in association with breakdown of the blood-brain barrier (BBB) with trafficking of activated $\mathrm{T}$ lymphocytes and other immune mediators, leading to acute tissue damage principally with breakdown of myelin but also with a degree of axonal destruction. ${ }^{1}$ These acute inflammatory lesions,

Address correspondence and reprint requests to David H. Miller, Multiple Sclerosis NMR Research Unit, Department of Neuroinflammation, Institute of Neurology, Queen Square, London WC1N 3BG, UK. E-mail: d.miller@ion.ucl.ac.uk. when they occur in clinically eloquent locations, lead to acute relapses of neurological deficit largely as a result of conduction block due to loss of myelin. There is usually good recovery from such relapses as a result of several mechanisms: resolution of inflammation, remyelination, restoration of conduction in persistently demyelinated fibers, and cortical adaptation. The onset with relapses and remissions (relapsing remitting MS) is seen in 85\% of cases.

In $15 \%$ of patients, the onset of MS is one of progressively increasing and irreversible disability (primary progressive MS). After a variable number of years this clinical pattern also emerges for $60 \%$ of those who started with relapsing remitting disease (secondary progressive MS). The progressive phase of MS has a poorer prognosis and there is compelling evidence- both from neuropathological observation ${ }^{2}$ and the application of magnetic resonance (MR) surrogate markers-that axonal loss is the main pathological substrate for such an evolution.

It is not well understood what mechanisms lead to chronic and extensive axonal loss. Although some axonal 
loss is attributable to the immediate effects of acute inflammation, this alone is not a sufficient explanation. Some patients, especially with primary progressive MS, have experienced few or no relapses and have little evidence on MRI scans for the past or present occurrence of inflammatory lesions. ${ }^{3}$ On the other hand, pathological and quantitative MR imaging (MRI) evidence has emerged that shows that the disease involves "normal appearing" white matter (NAWM) and normal appearing gray matter (NAGM). In NAWM, there is evidence of astrocytosis, microglial activation, perivascular inflammation and axonal loss. ${ }^{4,5}$ In NAGM, careful examination has revealed an abundance of demyelinated lesions that typically exhibit less inflammation than those seen in white matter but that may exhibit neuronal and axonal loss. ${ }^{6,7}$ Given the widespread nature of pathological changes in NAWM and NAGM, it seems likely that they contribute to clinical progression; how they relate to focal inflammatory demyelinating white matter lesions and to the overall pathogenesis of the disease is unclear.

\section{THE NEED FOR SURROGATE TOOLS TO MONITOR TREATMENTS IN MS}

It is difficult to perform definitive clinical trials in MS. The course is unpredictable and subject to short-term fluctuations, yet the development of irreversible disability often takes many years. The clinical scales commonly used for quantifying disability [e.g., the expanded disability status scale (EDSS)] have limited sensitivity and reproducibility and do not capture all of the patients' functional problems. Clinical assessments have an intrinsic subjective component and are prone to bias if either the patient or the investigator is unblinded. Definitive phase 3 trials with a clinical endpoint-either relapse rate or sustained increase in disability-require the participation of large patient numbers (usually several hundred), followed over at least 2 or 3 years, with a control versus active treatment parallel groups design and with double blinding. The trials are very expensive, cumbersome, and are slow in providing answers. Even so, they leave unanswered key questions such as: will treatment effects be sustained over much longer periods bearing in mind that MS usually evolves it full course over several decades?

Clearly, surrogate outcome measures would be very useful if they allowed for such large clinical trials to be avoided. To be a reliable surrogate measure, the outcome should be sensitive to disease evolution such that it provides an answer on therapeutic effect quickly and in small numbers of subjects. Most importantly, it should reflect and predict an important clinical outcome. In MS this outcome is either of the following: 1) reduction in relapse rate or 2) slowing the accumulation of irreversible disability. In view of the pathological basis of these clinical features it follows that biological surrogates proposed for MS fall broadly into two categories: 1) inflammatory white matter lesions (which cause relapses), and 2) neurodegenerative changes in lesions, NAWM, or NAGM (which cause progressive disability).

The rest of this review will discuss what has been learned using surrogate measures in MS. Because MR outcomes have been far and away the most useful and widely investigated outcomes, they will be the principal focus, although brief comments on other approaches will also be made where appropriate.

\section{MS BIOMARKERS OF INFLAMMATION}

\section{Body fluids}

Numerous studies have investigated the relationship of immunologically based measures obtained from serum, urine, or cerebrospinal fluid (CSF) with clinical evidence of disease activity and progression in MS. These studies have investigated T-cell subtypes (including activated $\mathrm{T}$-cell clones that are responsive to myelin antigens), soluble mediators, or products of inflammation [e.g., tumor necrosis factor (TNF)- $\alpha$, interleukins, and neopterin $\left.^{8}\right]$ and autoantibodies. Very recently, the presence of serum antibodies to myelin peptides has been reported to predict conversion from a clinically isolated syndrome to clinically definite MS. ${ }^{9}$ However, in general a consistent pattern of association with disease activity has not emerged; initially promising results from one laboratory have usually not been replicated. Serum and urine markers are liable to be diluted by noise because of systemic biological factors unrelated to the primary disease. Although CSF examination gets closer to the pathological process, methodological standardization is often difficult and it is not easy to obtain consent for lumbar puncture for research purposes only.

\section{Positron emission tomography: PK11195}

PK11195 is a radioligand detectable using positron emission tomography, which attaches to peripheral benzodiazepine receptors. These are expressed on activated microglial cells, which in turn have potentially important roles in MS as CNS-based antigen presenting cells. Increased PK11195 uptake has been detected in MS lesions and NAWM and deep gray matter nuclei. ${ }^{10,11}$ Given the cell specificity of the marker and potential importance of microglia in CNS inflammation it is surprising that more extensive clinical studies in MS have not been performed. This may reflect practical and methodological difficulties associated with quantitative PK11195 imaging and the limited opportunities to perform serial studies to avoid cumulative exposure to a radioactive compound.

\section{MRI}

Gadolinium enhancing and T2 lesions. Most gadolinium $(\mathrm{Gd})$ enhancing and T2-weighted lesions are clin- 
TABLE 1. Therapies which Reduce the Frequency of Relapses and New Gd Enhancing Lesions

- $\beta$-interferon $1 \mathrm{~b}$

- $\beta$-interferon 1a (SC)

- $\beta$-interferon 1a (IM)

- Glatiramer acetate

- Mitoxantrone

- Natalizumab

- Campath-1H

- Cladribine

ically silent; thus MRI has become an important tool for supporting an early and accurate diagnosis of MS in many patients. ${ }^{12}$ Postmortem and biopsy studies have demonstrated that inflammatory features-perivascular cuffs of lymphocytes and macrophage infiltrates-correlate with Gd enhancement in MS lesions. ${ }^{13,14}$ Enhancement is consistently seen in new brain lesions in relapsing remitting and secondary progressive MS and usually lasts 2-6 weeks, similar to the duration of a relapse. Enhancing lesions are more often seen during relapse than remission. ${ }^{15}$ Although the majority are asymptomatic, enhancing cord lesions are more likely to result in clinical relapse when they occur in a clinically eloquent location, e.g., spinal cord ${ }^{16}$ or optic nerves. ${ }^{17}$ In optic neuritis, enhancement of the symptomatic lesion correlates with acute visual loss and conduction block (reduced amplitude of the visually evoked potential). ${ }^{18}$ Such observations and a subsequent meta-analysis of nine studies ${ }^{19}$ suggest that Gd enhancement is a reasonable surrogate marker for acute relapses. This view is supported by the concordant effect of numerous therapies in reducing both enhancing lesions and relapses (Table 1). Of particular theoretical interest is the demonstration that an antiadhesion molecule which specifically blocks trafficking of T lymphocytes across the BBB has been shown to reduce both enhancing lesions and relapses in a 6-month phase 2 study. ${ }^{20}$

T2-weighted scans are sensitive in detecting focal MS white matter lesions. Although they are pathologically nonspecific, serial studies in relapsing remitting MS at weekly intervals have shown that almost all new T2 lesions begin as a region of Gd enhancement, thus indicating an initial inflammatory phase in their evolution. Therefore, the total load of T2 lesions observed in early relapsing remitting MS provides an indication of the amount of inflammation that has occurred to date. The same cannot be said of more advanced disease and primary progressive MS, in which there is evidence that at least some of the T2 abnormalities develop independently of BBB breakdown. ${ }^{3}$

Whereas Gd enhancing and T2 lesions can be related to relapses they correlate poorly, if at all, with the evolution of disability. ${ }^{19}$ It is notable, for example, that patients with primary progressive MS often have few Gd enhancing lesions and small T2 lesion loads. ${ }^{3}$ One exception to this rule is that the accumulation of inflammatory lesions during the first 5 years of relapse-onset MS (measured as total T2 volume) is partly related to current and future disability. ${ }^{21}$ These imaging observations fit broadly with the observation that relapse rate in the early years of relapsing remitting MS has an influence on the time-to-develop of moderate disability (EDSS 4$)^{22}$ but not the subsequent evolution to more severe disability. ${ }^{23}$ Taken together, early clinical (relapses) and imaging (Gd enhancing/T2 lesions) features of inflammation may have a modest impact on long-term disability but later inflammation probably does not.

In relapsing remitting MS, monthly standard dose $(0.1$ $\mathrm{mmol} / \mathrm{kg}$ ) Gd-enhanced T1-weighted brain MRI reveals $\sim 10$ new $\mathrm{Gd}$ enhancing lesions for every clinical relapse. ${ }^{24,25}$ Substantial activity is also found in secondary progressive MS, mainly among those having superimposed relapses. ${ }^{26}$ The number of Gd enhancing lesions is increased by weekly scanning, ${ }^{27}$ spinal imaging, ${ }^{16}$ tripledose $\mathrm{Gd}(0.3 \mathrm{mmol} / \mathrm{kg}),{ }^{28}$ magnetization transfer T1weighted sequences, ${ }^{29}$ delayed scanning, ${ }^{30}$ and thinner slices. In practice, such approaches are rarely used in clinical trials and have little impact on sample size requirements for trials because variability between patients also increases. ${ }^{31}$

In relapsing remitting MS, a parallel groups design with a placebo arm requires $\sim 2 \times 40$ patients to show a $60 \%$ reduction in new enhancing lesions over 6 months, ${ }^{32}$ with a 1 -month run in scan reducing sample sizes by $\sim 30 \% .{ }^{33}$ Such a trial design has become the standard way to evaluate efficacy of new anti-inflammatory therapies in phase 2 trials. A positive result provides the basis for proceeding to a phase 3 trial that will have a primary clinical endpoint and therefore be larger, longer, and more costly. Crossover phase 2 MRI outcome designs are more powerful than parallel group studies, because there is less intrapatient than interpatient variability in MRI activity, but may be complicated by regression to the mean.

The number and volume of $\mathrm{Gd}$ enhancing and T2weighted lesions has often been used during the last decade as a secondary or tertiary outcome measure in phase 3 clinical trials of disease modifying treatments in MS, in which the primary outcome has been a clinical endpoint, either slowing of accumulation of persistent disability or reduction of relapse rate. The positive effects of therapies on such MRI outcomes have been included in submissions to regulatory authorities in North America and Europe. The first example in which this occurred was a North American randomized trial of $\beta$-interferon $1 \mathrm{~b}$ in relapsing remitting MS, which was considered by the United States Food and Drug Administration in 1993 and in which therapy was associated 
with a striking reduction in the number of new T2 lesions. ${ }^{34}$ Although T2 and Gd enhancing lesion outcomes have provided supportive evidence of treatment benefit, their value has been limited by the generally modest correlation with clinical outcome, especially disability. This limitation has been evident both for natural history evolution and for extent of treatment response. MRI lesion outcomes have therefore not been regarded by either regulatory authorities or expert investigators ${ }^{35}$ as adequate to serve as primary outcome measures in definitive phase 3 trials.

MR spectroscopy. Using proton MR spectroscopy, an elevation of the peak for choline (Cho)-containing compounds is observed in acute MS lesions and reflects increased membrane turnover as a result of inflammation and/or myelin breakdown. Peaks at 0.9 and $1.3 \mathrm{ppm}$ are also seen in acute lesions and resolve over several months ${ }^{36}$; these are attributed to lipid breakdown products in association with demyelination

An increase in myoinositol (Ins) is seen in MS lesions and is more marked in T1 hypointense lesions. ${ }^{37}$ It is also observed in NAWM, in which the elevation has been correlated with disability in MS patients with both short and long disease durations. ${ }^{38,39}$ Ins is produced by glial cells and is a potential marker of astrocytosis, gliosis, or possibly microglial activation. Although Ins is difficult to quantify because of having a short T2 relaxation time (thus requiring MR spectroscopy with a short echo time), these interesting observations-suggesting it may detect a diffuse glial/inflammatory process associated with disease progression-should encourage further investigation of Ins in therapeutic trials.

Ultrasmall particles of iron oxide. A recently developed contrast agent uses ultrasmall particles of iron oxide (USPIO) which uptake in macrophages, thus providing an opportunity to detect MS lesions containing this cell type. Given the potential for macrophages to induce myelin or even axonal destruction in MS this appears to be a promising tool. To date, only one small study of MS patients has been reported. In this group, USPIO enhancement was seen in a subset of about $50 \%$ of $\mathrm{Gd}$ enhancing lesions. ${ }^{40}$

\section{MS BIOMARKERS OF NEURODEGENERATION}

\section{Body fluids}

Proposed markers of axonal breakdown have been studied in MS, most notably in the CSF. Recent work has identified a link between increasing dephosphorylation of CSF neurofilaments and increasing disability in MS. ${ }^{41}$ Other axonal markers include S100b and the 14-3-3 protein but no consistent relationship with the course of MS has yet emerged. The understandable reluctance of pa- tients to undergo serial lumbar puncture militates against using a CSF outcome measure in a clinical trial.

\section{MR markers}

\section{Atrophy measures}

Of all the proposed MR measures of the neurodegenerative component of MS, measurement of tissue loss (atrophy) is the most attractive and robust and to date is the most widely used in treatment trials. Axons make the largest bulk contribution to white matter volume (45\%), followed by myelin (25\%) and other tissue elements (glial and vascular tissues and water). ${ }^{42}$ Neuronal cell bodies and axons contribute the bulk of gray matter volume although there is also myelin in gray matter, albeit to a lesser extent than in white matter. It follows that atrophy of white or gray matter in MS likely reflects axonal and neuronal loss. In a study of the spinal cord of five MS cases, atrophy and axonal loss were studied. ${ }^{43}$ There was axonal loss within spinal cord lesions of between $45 \%$ and $85 \%$. Atrophy was more marked in the cervical than the lumbar area and affected gray and white matter equally.

Axonal loss is not the sole cause of atrophy and loss of myelin per se will contribute. Variation in glial bulk, inflammation, and tissue water content will also affect global or regional volume measures in MS: acute inflammation and gliosis will increase volume whereas decreased tissue water and inflammation due to treatment, dehydration, or other factors, will decrease volume. It is likely that the use of atrophy to measure progressive neurodegeneration in MS will be made less sensitive because of the volumetric fluctuations attributable to inflammation. It should also be kept in mind that antiinflammatory therapies (e.g., high-dose corticosteroids or $\beta$-interferon) may reduce brain volume without there having been axonal loss. If such an effect is anticipated, it would seem wise to allow a period of time after receiving such therapy for the anti-inflammatory volume reduction effect to have occurred before using ongoing atrophy as a presumed measure of axonal loss.

The optimal technique for detecting atrophy should be reproducible, sensitive to change, accurate, and practical to implement, although small errors of accuracy are probably insignificant, as long as they are constant between subjects and over time. The two distinct methodological aspects involved in measuring tissue volumes are data acquisition and data analysis.

Data acquisition. The ability to reduce partial volume errors with high-resolution scans means that 3-D acquisitions are attractive, although 2-D sequences have also been used successfully to derive volume measures in the CNS. ${ }^{44}$ For whole-brain atrophy measurements segmentation of the brain is necessary, and suppression of CSF helps to generate a sharp distinction in signal between cerebral and extracerebral matter. The most widely used 
3-D sequence is a T1-weighted gradient echo, with or without added CSF suppression, the latter provided by an inversion recovery prepulse, which allows $1 \times 1 \times 1$ $\mathrm{mm}$ resolution. Specific study of white or gray matter requires good contrast between white matter, gray matter, CSF, and lesions and may be aided by multiplecontrast acquisitions, e.g., $\mathrm{T}_{1}, \mathrm{~T}_{2}$ and proton density.

Data analysis methods. Manual outlining or linear measurements provide the simplest approach to measuring changes in volume. An experienced observer is required who is familiar with normal neuroanatomy and pathology. Manual segmentation is useful in small structures or regions, e.g., third ventricle, where significant atrophy is reported in MS. ${ }^{45}$ Disadvantages of manual segmentation include operator bias, long analysis time and poor reproducibility when compared with automated techniques.

Semi-automated methods improve speed and reproducibility. Regional segmentation algorithms, e.g., seed growing, ${ }^{46}$ contouring outline of lesions, ${ }^{47}$ spinal cord, optic nerves, and ventricles. ${ }^{48,49}$ Measurement reproducibility improves from $\sim 3-5 \%$ for manual outlining to $\sim 1-3 \%$ for semiautomated approaches for measuring spinal cord and ventricles.

Many automated methods exist for segmentation (and thus volume measurement) of the whole brain. Both single contrast ${ }^{50}$ and multispectral data ${ }^{51,52}$ have been used for whole-brain segmentation. Usually the difference in signal intensity between brain parenchyma and CSF on a single-contrast acquisition is enough to drive the segmentation process. Segmentation of gray and white matter may also be accomplished with either single-contrast or multispectral data, although additional sophistication is required to separate the two tissue types. Methods include statistical parametric mapping-based segmentation $^{53}$ and the fuzzy C-means algorithm. ${ }^{54}$ Masking of MS lesions is necessary to avoid their misclassification.

Because atrophy is the measurement of change in volume, measurements of absolute volumes at separate time points are not necessarily needed; information may be obtained by looking for differences between serial scans. ${ }^{55}$ Nonlinear registration of such scans produces deformation fields that yield information concerning regional and global atrophy. ${ }^{56}$ Rigid body registration can be used to track the displacement of the surface of the brain during atrophy. Using the inner table of the skull as standard of reference, Freeborough and Fox ${ }^{57}$ have developed a method to quantify atrophy with high sensitivity in many neurological diseases.

Comparisons between groups of patients are confounded by the presence of substantial inter-subject variations in head size that can mask differences attributable to atrophy. Normalizing the brain volume to head size reduces these variations. Relative volumes also remove variability in volume data due to scanner instability. A number of normalization methods have emerged: the scalp, ${ }^{57}$ total intracranial capacity determined by the sum of the volumes of gray matter, white matter, and $\mathrm{CSF},{ }^{58,59}$ or the sum of the brain and ventricular and sulcal $\mathrm{CSF}^{60}$ have all been used to create volumes for normalization.

Clinically isolated syndromes. Many patients with clinically isolated syndromes (CIS) (i.e., a single relapse of the sort seen in MS) affecting the optic nerve, brain stem, or spinal cord already have disseminated cerebral white matter lesions typical of MS and develop clinically definite MS with follow-up. ${ }^{61,62}$ A cohort of 55 CIS patients ${ }^{63}$ has been followed over a 1 -year period to investigate ventricular enlargement. Significant enlargement was seen in the 18 patients who developed clinical MS (i.e., experienced a second clinical relapse), and in the 40 patients with abnormal brain MRI at presentation. There was no change in ventricular volume in the 15 patients with normal imaging. Thus, brain atrophy occurs at the earliest clinical stage of MS. Atrophy of about $10-15 \%$ has also been observed in the optic nerve following a single attack of optic neuritis. ${ }^{64}$

Relapsing remitting MS. Recent studies have shown that brain atrophy occurs in relapsing remitting MS even within 3 years of symptom onset. ${ }^{58}$ Both white and gray matter atrophy are observed. ${ }^{58,65}$ Using a whole brain ratio (WBR) method, brain atrophy was assessed over two years in a placebo-controlled trial of beta interferon in relapsing remitting MS. ${ }^{66}$ Atrophy measures were available in 519 patients, 172 of whom were placebotreated. Significant brain atrophy was seen in the total cohort over 2 years; the mean WBR decreased by $1.4 \%$. The baseline WBR was weakly correlated with T2 lesion load. No difference in the rate of atrophy was seen between treatment arms.

Cerebral atrophy has been evaluated from 52 relapsing remitting patients for 6 months before and 24 months following $\beta$-interferon treatment and correlated with other MRI lesion and clinical parameters. ${ }^{67}$ During the 2 years of treatment there was a significant reduction of brain volume (mean $-2.2 \%$ ) that correlated weakly with the mean number of enhancing lesions on monthly scans during the 6 months pre-treatment. During the 2 years of treatment, 26 patients exhibited significant atrophy and 26 did not; in the former group, 13 experienced an increase in disability whereas in the latter group only 3 became more disabled. This confirms other studies by showing a link between increasing atrophy and disability. ${ }^{68,69}$

In a 2-year placebo-controlled trial of $\beta$-interferon in relapsing remitting MS, atrophy was measured from yearly scans using brain parenchymal fraction (BPF). The mean BPF decrease was similar in both arms in year 1 , but was smaller in the $\beta$-interferon arm in year $2 .^{60}$ 
The changes in BPF during this 2-year period showed little or no correlation with lesion measures. Prolonged 8 -year follow-up of some of the placebo cohort from this trial assessed the longer term relationship between earlier $\mathrm{BPF}$ change and later disability. ${ }^{70}$ Comparison of patient quartiles based on change in BPF over the first 2 years revealed a greater likelihood of developing severe disability (EDSS of 6 or more at follow-up) in those with the most atrophy during the initial 2 years.

A 9-month placebo-controlled trial of glatiramer acetate in 239 relapsing remitting MS cases revealed a mean $0.7-0.8 \%$ reduction in central cerebral volume with no significant differences between the patient groups. ${ }^{71}$ The study showed a weak association between enhancing lesion numbers and atrophy.

Progressive forms of MS. Atrophy is seen in both the brain and spinal cord in secondary and primary progressive MS. The most marked atrophy occurs in secondary progressive disease and correlates with disability. ${ }^{45,72,73}$ In primary progressive MS, significant atrophy of brain and cord over 1 year was evident in a large cohort of primary progressive patients drawn from 6 European centers. ${ }^{74}$ Change in cerebral volume over 1 year correlated only weakly with change in $\mathrm{T} 1$ and $\mathrm{T} 2$ brain load. More recently, progressive cerebral and cervical cord atrophy has been observed over 5 years of follow-up in a cohort of 41 primary progressive MS patients. ${ }^{75}$ The rates of atrophy appeared to be relatively constant within individual patients but varied between subjects.

A study of 16 patients with primary progressive MS evaluated riluzole, a neuroprotective glutamate antagonist, using change in cord area as a putative measure of progressive axonal loss. ${ }^{76}$ During 1 year pretreatment, there was a $2 \%$ reduction in mean cord area whereas during 1 year on treatment the cord area was stable (mean decrease of $0.2 \%$ only), but the difference was not significant. This preliminary study indicates the potential of using tissue volume measures in larger cohorts to study the efficacy of neuroprotective agents.

Therapeutic trials have evaluated the effect of three immunomodulatory agents in secondary progressive MS: $\beta$-interferon, ${ }^{44}$ Campath- $1 \mathrm{H},{ }^{77}$ and cladribine. ${ }^{78,79}$ In spite of all three therapies suppressing inflammatory MRI lesions, there was no evidence for a significant slowing in the rate of ongoing cerebral atrophy. In the $\beta$-interferon trial there was $\sim 1 \%$ loss of central cerebral volume per year in the treated and placebo arms.

Time-course issues for measuring atrophy. Although it is clear that significant tissue loss can be detected in MS within as little as 12 months, little work has been done to determine the optimal sample sizes and length of study required to demonstrate significant slowing of progressive atrophy as a result of therapeutic intervention. This is a priority for further research, which should include a consideration of the stage of disease (e.g., relapsing remitting, secondary progressive), type of acquisition and image analysis method, region of CNS being studied (e.g., whole brain, regional brain, spinal cord, etc.), frequency of scanning, and other potential confounding factors (e.g., atrophy due to anti-inflammatory therapy).

Summary of atrophy as a surrogate marker in MS. For the following reasons, atrophy has emerged as a preferred method for monitoring the neurodegenerative process in MS: 1) robust methods for detecting tissue loss are available, 2) it is progressive from onset and increases with increasing disability, 3 ) it correlates only modestly with inflammatory lesions, thus providing additional information in therapeutic monitoring, and 4) whereas a number of existing therapies have shown good suppression of inflammatory lesions, an effect on progressive atrophy has been less evident. A recent review discusses in depth the methodological and clinical aspects of atrophy in MS. ${ }^{42}$

\section{MR spectroscopy: $N$-acetyl aspartate}

The main peak in the proton MR spectrum from human adult CNS is $\mathrm{N}$-acetyl aspartate (NAA), an amino acid contained almost exclusively in neurones and axons. A reduction in NAA provides evidence of axonal dysfunction or loss and has been consistently reported in MS lesions and NAWM. ${ }^{80}$ A greater reduction of NAWM NAA is observed in secondary and primary progressive than in relapsing remitting MS, ${ }^{80,81}$ and disability has been correlated with reduced NAA in both cerebral ${ }^{82,83}$ and cerebellar ${ }^{84}$ NAWM. Decreased NAA has also been observed in cortical gray matter in early relapsing remitting MS, suggesting that early neuronal cell body damage is occurring. ${ }^{38}$ It is reduced by $\sim 20 \%$ in thalamic gray matter in secondary progressive MS and in a postmortem study the decrease in NAA (accompanied by atrophy) was associated with decreased numbers of neurones. $^{7}$ In primary progressive MS, reduction of NAA and atrophy appear to be relatively independent of T2 lesion load. ${ }^{85}$

Two approaches have been used to measure NAA: 1) an absolute measure of concentration using and external standard reference of known concentration, and 2) a ratio of NAA/Cr which assumes that $\mathrm{Cr}$ (creatine/phosphocreatine) remains stable in pathological situations. Although both approaches have produced robust evidence that NAA is reduced in MS lesions and normal appearing tissues, abnormalities of $\mathrm{Cr}$ may also occur. Therefore, absolute measures are preferable. A methodological approach of recent interest is the quantitation of wholebrain NAA. ${ }^{86}$ This has been reported to be low in patients with CIS. ${ }^{87}$ As a global marker of the progressive neurodegenerative process in MS it appears promising, although any changes observed are not anatomically localized and could represent abnormality in lesions, 
NAWM, or gray matter. The resonance for whole-brain NAA is broad and requires manual delineation for quantification; its analysis is potentially subject to bias and poor reproducibility. In contrast, the narrow NAA resonances from small voxels, obtained as a single region or as part of a spectroscopic imaging slice, can be automatically identified and quantified with a model that uses as reference a solution with a known concentration of NAA. ${ }^{88}$

A limitation of spectroscopy is the low signal-to-noise ratio and modest reproducibility of the measured metabolite concentrations. For this reason, it has been little used in multicenter therapeutic trials. Two small singlecenter studies of patients treated with $\beta$-interferon have produced conflicting results. One study showed an increase in NAA, suggesting a therapy-induced reversal of axonal dysfunction. ${ }^{89}$ The other showed a decrease in NAA, suggesting that progressive axonal loss continues in spite of treatment. ${ }^{90}$ In spite of methodological difficulties, more vigorous efforts to investigate NAA as a surrogate outcome in trials of neuroprotection in MS would seem warranted, given that this metabolite provides specific information on axonal survival and function.

\section{Diffusion tensor imaging}

Diffusion tensor imaging offers a potentially more specific investigation of the integrity of white matter tracts. Fractional anisotropy (FA) indicates the orientation of diffusion and is high along well-defined pathways such as the corpus callosum, pyramidal tracts, and optic radiations. A reduction in FA in such pathways is therefore a potential marker of axonal structural integrity. Most recently, algorithms have been developed for identifying individual white matter tracts. Diffusion tractography can be performed using several approaches. ${ }^{91}$ Problems arise in areas of crossing tracts or in which there are sharp bends in a tract. However, using tractography algorithms it is now possible to quantify the size and FA of major pathways in the brain such as optic radiation and pyramidal tract. ${ }^{92}$

\section{T1 hypointense lesions}

About 20-30\% of T2 lesions appear hypointense on T1-weighted MRI. In serial studies, some of these socalled "black holes" are reversible, first appearing during the phase of acute inflammation with Gd enhancement and resolving over subsequent months. Such a course reflects initial edema and demyelination with subsequent resolution of edema and remyelination..$^{93}$ Other lesions persist as chronic stable "black holes" and these have been shown to have greater axonal loss than those T2 lesions which are $\mathrm{T} 1$ isointense. ${ }^{94}$ There is a strong correlation between $\mathrm{T} 2$ and $\mathrm{T} 1$ hypointense lesion loads ${ }^{95}$ and the latter adds little to the former when monitoring treatment effect on total lesion load. It is useful to mon- itor the evolution of individual Gd enhancing lesions into permanent black holes. A reduction in such evolution can be considered as the surrogate marker for improved recovery from acute relapses (i.e., there is less residual axonal loss due to acute inflammation). Such a treatment effect has been demonstrated for glatiramer acetate ${ }^{96}$ and natalizumab ${ }^{97}$ but not $\beta$-interferon. ${ }^{98}$

\section{Other measures}

Many other quantitative MR measures have been applied to study MS, e.g., magnetization transfer ratio (MTR), T1 relaxation time, and the apparent diffusion coefficient. Such measures are sensitive in depicting subtle abnormalities in NAWM and NAGM and convincing evidence has emerged that increasing abnormality in these tissues is associated with clinical progression.99,100 However, these subtle MR changes are pathologically nonspecific and could potentially represent the effects of inflammation, gliosis, or axonal loss, all of which occur in NAWM. ${ }^{4,101}$ Although they may be valuable for monitoring clinically relevant disease progression they should not be considered as specific markers of neurodegeneration. Another abnormality reported in MS is a decrease in signal on T2-weighted images of deep gray matter structures, ${ }^{102}$ which probably reflects increased iron deposition; whether or not this correlates with neuronal loss is unclear.

\section{SUMMARY AND RECOMMENDATIONS}

The foregoing assessment of MS biomarkers can be summarized into two key points. First, of the various approaches that have been considered, the only methods that are currently used on a regular basis as outcome measures in treatment trials are those derived from MR; the recommendations for surrogate outcome measures for MS clinical trials are accordingly confined to MR outcomes. Second, a broad concept has emerged which characterizes MS into two main pathological processes or phases: 1) multifocal inflammation, and 2) diffuse neuroaxonal degeneration (FIG. 1).

The process of multifocal inflammation is most prominent in early relapsing remitting MS and is characterized clinically by relapses and on MRI by the development of focal new Gd enhancing and $\mathrm{T} 2$ lesions. In addition to demyelination, a degree of acute axonal injury and loss occurs because of this acute inflammation and may be inferred from the evolution to a permanent $\mathrm{T} 1$ hypointense lesion or the development of a permanent decrease in NAA. Focal atrophy from individual lesions is not readily appreciated in the brain but can be measured in the anatomically isolated optic nerve and averages $\sim 10$ $15 \%$ after an attack of optic neuritis. If the aim of a new experimental therapy is to prevent relapses, new Gd enhancing and $\mathrm{T} 2$ lesions can be regarded as an appropriate 


\section{Clinical course and pathogenic mechanisms in Multiple Sclerosis}

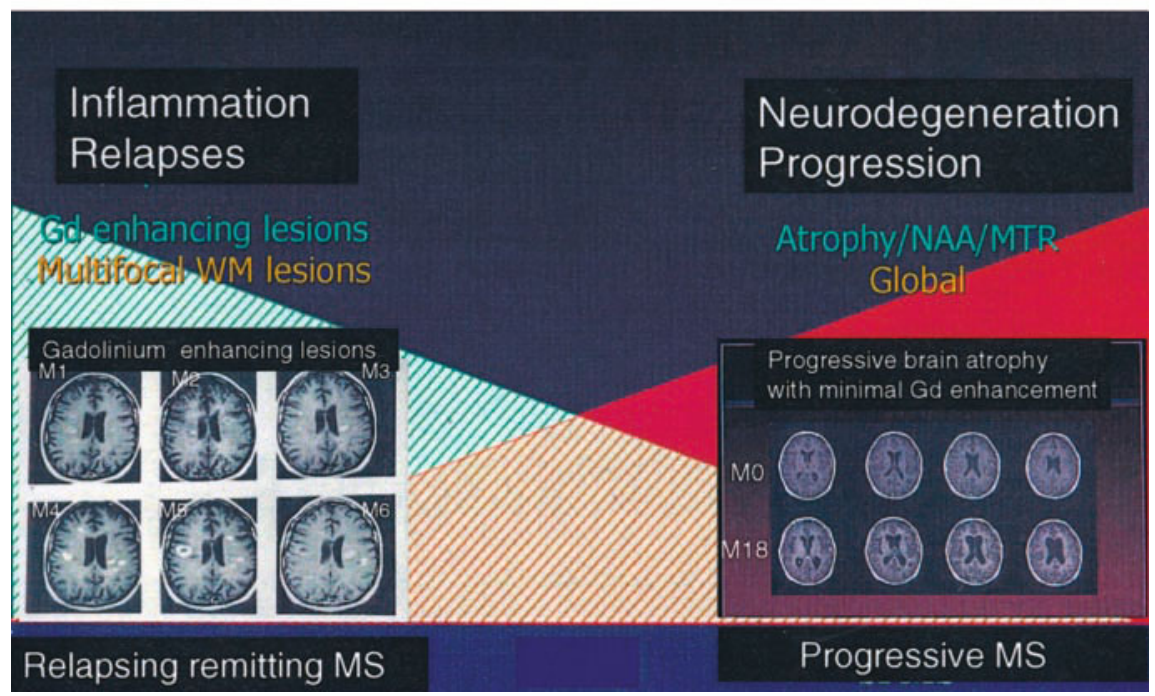

FIG. 1. This dual pathogenic model of MS proposes an early phase predominated by multifocal inflammation and relapses and a later phase by progressive neuroaxonal loss and increasing disability.

surrogate outcome measure. The number of new lesions can be considered as the imaging equivalent of relapse frequency, whereas their evolution to T1 hypointense lesions might be regarded as the equivalent to the development of persistent disability following a relapse. However, apart from the early years of relapsing remitting MS (and then only to a moderate extent), new lesions are not reliable in predicting future disability and should not be considered as a surrogate for that clinical outcome.

The process of diffuse neuroaxonal degeneration involves white matter lesions, NAWM and gray matter and is increasingly prominent with increasing disability and the progressive phase of MS. The two most specific MR methods for detecting neuroaxonal loss are atrophy and decreased NAA. The former has been preferred in clinical trials because more reproducible and sensitive methods are available to detect it. Although other MR markers of diffuse disease are not specific for axonal loss (e.g., MTR, T1, diffusion coefficient), they do provide, along with atrophy, a sensitive measure of a diffuse, progressive underlying process that appears relevant to clinical progression.

Both atrophy and intrinsic NAWM and NAGM abnormalities are present from the earliest clinical stages of disease. They are at least in part independent of focal lesions but may also be partly related, by providing a trigger for or being a consequence of focal inflammation (the latter, by causing axonal transection, leads to secondary Wallerian degeneration in NAWM). Although not yet confirmed, the potential importance of atrophy, NAWM, and NAGM measures for predicting future disability is readily apparent. It is therefore recommended that atrophy be measured in trials aiming to prevent disability at all stages of disease (CIS, relapsing remitting, primary, and secondary progressive) and where feasible, NAA should also be measured along with other techniques to monitor progressive NAWM and NAGM abnormality (e.g., MTR).

Finally, it is important to remember that although holding much promise, the MR surrogates for neuroaxonal loss and diffuse disease are not yet validated as predicting future disability. Long-term follow-up studies from near onset will be needed to establish this relationship. In the meantime, definitive clinical trials should continue to measure an appropriate disability endpoint.

Acknowledgments: I thank all of my colleagues in the MS NMR Research Unit for their excellent work and collaboration, and the MS Society of Great Britain and Northern Ireland for their continued support of the Unit.

\section{REFERENCES}

1. Trapp BD, Peterson J, Ransohoff RM, Rudick R, Mork S, Bo L. Axonal transection in the lesions of multiple sclerosis. $N$ Engl $J$ Med 338:278-285, 1998.

2. Lassmann H, Suchanek G, Ozawa K. Histopathology and the blood-cerebrospinal fluid barrier in multiple sclerosis. Ann Neurol 36(Suppl):S42-S46, 1994.

3. Thompson A, Kermode A, MacManus D, Kendall B, Kingsley D, Moseley I, McDonald W. Major differences in the dynamics of primary and secondary progressive multiple sclerosis. Ann Neurol 29:53-62, 1991.

4. Allen IV, McKeown SR. A histological, histochemical and biochemical study of the macroscopically normal white matter in multiple sclerosis. J Neurol Sci 41:81-89, 1979.

5. Evangelou N, Esiri MM, Smith S, Palace J, Mathews PM. Quantitative pathological evidence for axonal loss in normal appearing white matter in multiple sclerosis. Ann Neurol 47:391-395, 2000. 
6. Peterson JW, Bo L, Mork S, Chang A, Trapp BD. Transected neuritis, apoptotic neurons, and reduced inflammation in cortical multiple sclerosis lesions. Ann Neurol 50:389-400, 2001.

7. Cifelli A, Arridge M, Jezzard P, Esiri MM, Palace J, Matthews PM. Thalamic neurodegeneration in multiple sclerosis. Ann Neurol 52:650-653, 2002.

8. Giovannoni G, Lai M, Kidd D, Thorpe JW, Miller DH, Thompson AJ, Keir G, Feldmann M, Thompson EJ. Daily urinary neopterin excretion as an immunological marker of disease activity in multiple sclerosis. Brain 120:1-13, 1997.

9. Berger T, Rubner N, Schautzer F, Egg R, Ulmer H, Mayringer I et al. Antimyelion antibodies as a predictor of clinically definite multiple sclerosis after a first demyelinating event. $N$ Engl $J$ Med 349:139-145, 2003.

10. Banati RB, Newcombe J, Gunn RN, Cagnin A, Turkheimer F, Heppner F et al. The peripheral benzodiazepine binding site in the brain in multiple sclerosis. Quantitative in vivo imaging of microglia as a measure of disease activity. Brain 123:2321-2337, 2000.

11. Debruyne JC, Versijpt J, van Laere KJ, de Vos F, Keppens J, Strijckmans $\mathrm{K}$ et al. PET visualization of microglia in multiple sclerosis patients using [11C]PK11195. Eur J Neurol 10:257264, 2003.

12. McDonald WI, Compston A, Edan G, Goodkin D, Hartung H-P, Lublin FD et al. Recommended diagnostic criteria for multiple sclerosis: guidelines from the international panel on the diagnosis of multiple sclerosis. Ann Neurol 50:121-127, 2001.

13. Katz D, Taubenberger J, Cannella B, McFarlin D, Raine C, $\mathrm{McF}$ arland $\mathrm{H}$. Correlation between magnetic resonance imaging findings and lesion development in multiple sclerosis. Ann Neurol 34:661-669, 1993.

14. Bruck W, Bitsch A, Kolenda H, Bruck Y, Stiefel M, Lassmann H. Inflammatory central nervous system demyelination: correlation of magnetic resonance imaging findings with lesion pathology. Ann Neurol 42:783-793, 1997.

15. Grossman RI, Gonzales-Scarano F, Atalas SW, Galetta S, Silberberg DH. Multiple sclerosis: gadolinium enhancement in MR imaging. Radiology 169:117-122, 1986.

16. Thorpe JW, Kidd D, Moseley IF, Kendall BE, Thompson AJ, MacManus DG et al. Serial gadolinium enhanced MRI of the brain and spinal cord in early relapsing-remitting multiple sclerosis. Neurology 46:373-378, 1996.

17. Hickman SJ, Toosy AT, Miszkiel KA, Jones SJ, Altmann DR, MacManus DG et al. Predicting visual recovery following optic neuritis: a clinical, electrophysiological and magnetic resonance imaging study. Mult Scler 9(Suppl 1):S95, 2003.

18. Youl BD, Turano G, Miller DH, Towell AD, MacManus DG, Moore SG et al. The pathophysiology of optic neuritis: an association of gadolinium leakage with clinical and electrophysiological deficits. Brain 114:2437-2450, 1991.

19. Kappos L, Moeri D, Radue EW, Schoetzau A, Schweikert K, Barkhof $\mathrm{F}$ et al. Predictive value of gadolinium-enhanced magnetic resonance imaging for relapse rate and changes in disability or impairment in multiple sclerosis: a meta-analysis. Lancet 353: 964-969, 1999.

20. Miller DH, Khan OA, Sheremata WA, Blumhardt LD, Rice G, Libonati LA, Wilmer-Hulme A, Dalton C, Miszkiel K, O’Connor PW. A controlled trial of natalizumab for relapsing multiple sclerosis. N Engl J Med 348:15-23, 2003.

21. Brex PA, Ciccarelli O, O'Riordan JI, Sailer M, Thompson AJ, Miller DH. A longitudinal study of abnormalities on MRI and disability from multiple sclerosis. $N$ Engl $J$ Med 346:158-164, 2002.

22. Confavreux C, Vukusic S, Adeleine P. Early clinical predictors and progression of irreversible disability in multiple sclerosis: an amnesic process. Brain 126:770-782, 2003.

23. Confavreux C, Vukusic S, Moreau T, Adeleine P. Relapses and progression of multiple sclerosis. $N$ Engl J Med 343:1430-1438, 2000.

24. Harris JO, Frank JA, Patronas N, McFarlin DE, McFarland HF. Serial gadolinium-enhanced magnetic resonance imaging scans in patients with early, relapsing-remitting multiple sclerosis: impli- cations for clinical trials and natural history. Ann Neurol 29:548 $555,1991$.

25. Thompson AJ, Miller DH, Youl B, MacManus D, Moore S, Kingsley D et al. Serial gadolinium enhanced MRI in relapsing remitting multiple sclerosis of varying disease duration. Neurology 42:60-63, 1992.

26. Tubridy N, Coles A, Molyneux P, Compston DAS, Barkhof F, Thompson AJ et al. Secondary progressive multiple sclerosis: the relationship between short-term MRI activity and clinical features. Brain 121:225-231, 1998.

27. Lai HM, Hodgson T, Gawne-Cain M, Webb S, MacManus D, McDonald WI et al. A preliminary study into the sensitivity of disease activity detection by serial weekly magnetic resonance imaging in multiple sclerosis. J Neurol Neurosurg Psychiatry 60:339-341, 1996.

28. Filippi M, Yousry T, Campi A, Kanziora C, Colombo B, Voltz R et al. Comparison of triple dose versus standard dose gadoliniumDTPA for detection of MRI enhancing lesions in patients with MS. Neurology 46:379-384, 1996.

29. van Waesberghe JHTM, Castelijns JA, Roser W, Silver N, Yousry T, Lycklama A, Nijeholt GJ et al. Single-dose gadolinium with magnetization transfer versus triple-dose gadolinium in the MR detection of multiple sclerosis lesions. AJNR Am J Neuroradiol 18:1279-1285, 1997.

30. Silver NC, Good CD, Barker GJ, MacManus DG, Thompson AJ, Moseley IF et al. Sensitivity of contrast enhanced MRI in MS effects of gadolinium dose, magnetization transfer contrast and delayed imaging. Brain 120:1149-1161, 1997.

31. Silver NC, Good CD, Sormani MP, MacManus DG, Thompson AJ, Filippi M, Miller DH. A modified protocol to improve the detection of enhancing brain and spinal cord lesions in multiple sclerosis. J Neurol 248:215-224, 2001.

32. McFarland HF, Frank J, Albert P, Smith M, Martin R, Harris J et al. Using gadolinium-enhanced magnetic resonance imaging lesions to monitor disease activity in multiple sclerosis. Ann Neurol 32:758-766, 1992.

33. Tubridy N, Ader HJ, Barkhof F, Thompson AJ, Miller DH. Exploratory treatment trials in multiple sclerosis using MRI: sample size calculations for relapsing remitting and secondary progressive subgroups using placebo controlled parallel groups. J Neurol Neurosurg Psychiatry 64:50-55, 1998.

34. Paty DW, Li DK. UBC MS/MRI Study Group and IFNB Multiple Sclerosis Study Group. Interferon-1b is effective in relapsingremitting multiple sclerosis. II. MRI analysis result of a multicenter, randomized, double-blind, placebo-controlled trial. $\mathrm{Neu}$ rology 43:662-667, 1993.

35. McFarland HF, Barkhof F, Antel J, Miller DH. The role of MRI as a surrogate outcome measure in multiple sclerosis. Mult Scler 8:40-51, 2002.

36. Davie C, Hawkins CP, Barker GJ, Brennan A, Tofts PS, Miller DH, McDonald WI. Serial proton magnetic resonance spectroscopy of multiple sclerosis lesions. Brain 117:49-58, 1994.

37. Brex PA, Parker GJM, Leary SM, Molyneux PD, Barker GJ, Davie CA et al. Lesion heterogeneity in MS: a study of the relations between appearances on $\mathrm{T} 1$ weighted images, T1 relaxation times and metabolite concentrations. J Neurol Neurosurg Psychiatry 68:627-632, 2000.

38. Chard D, Griffin CM, McLean MA, Kapellar P, Kapoor R, Thompson AJ, Miller DH. Brain metabolite changes in cortical grey matter and normal appearing white matter in clinically early relapsing remitting multiple sclerosis. Brain 125:2342-2352, 2002.

39. Kapeller P, Brex PA, Chard D, Dalton C, Griffin CM, McLean MA, Parker GJM, Thompson AJ, Miller DH. Quantitative $1 \mathrm{H}-\mathrm{MR}$ spectroscopic imaging 14 years after presenting with a clinically isolated syndrome suggestive of MS. Mult Scler 8:207210, 2002.

40. Dousset V, Brochet B, Cailee J-M, Petry K. MS lesions enhancement with ultrasmall particle iron oxide: the first phase II study. Rev Neurol 156(Suppl 3):3S40, 2000.

41. Petzold A, Eikelenbomm MJ, Keir G, Lazeron RC, Polman CH, Uitdehaag B et al. Dynamic of axonal pathology in multiple sclerosis. Mult Scler 8(Suppl 1):S25, 2002. 
42. Miller DH, Barkhof F, Frank JA, Parker GJM, Thompson AJ. Measurement of atrophy in multiple sclerosis: pathological basis, methodological aspects and clinical relevance. Brain 125:1676$1695,2002$.

43. Bjartmar C, Kidd G, Mork S, Rudick R, Trapp BD. Neurological disability correlates with spinal cord axonal loss and reduced $\mathrm{N}$-acetyl aspartate in chronic multiple sclerosis patients. Ann Neurol 48:893-901, 2000.

44. Molyneux PD, Kappos L, Polman C, Pozzilli C, Barkhof F, Filippi $\mathrm{M}$ et al. The effect of interferon $\beta$-1b treatment on MRI measures of cerebral atrophy in secondary progressive multiple sclerosis. Brain 123:2256-2263, 2000

45. Simon JH, Jacobs LD, Campion MK, Rudick RA, Cookfair DL, Herndon RM et al. A longitudinal study of brain atrophy in relapsing multiple sclerosis. Neurology 53:139-148, 1999.

46. Rovaris M, Inglese M, van Schijndel RA, Sormani MP, Rodegher M, Comi G, Filippi M. Sensitivity and reproducibility of volume change measurements of different brain portions on MR scans from patients with multiple sclerosis. J Neurol 247:960-965, 2001.

47. Plummer DL. DispImage: a display and analysis tool for medical images. Revista di Neuroradiologica 5:489-495, 1992.

48. Losseff NA, Webb SL, O'Riordan JI, Page R, Wang L, Barker GJ et al. Spinal cord atrophy and disability in multiple sclerosis. A new reproducible and sensitive MRI method with potential to monitor disease progression. Brain 119:701-708, 1996a.

49. Lycklama a Nijeholt GJ, van Walderveen MA, Castelijns JA, van Waesberghe JHTM, Polman C, Scheltens P et al. Brain and spinal cord abnormalities in multiple sclerosis. Correlation between MRI parameters, clinical subtypes and symptoms. Brain 121: 687-697, 1998.

50. Chard DT, Parker GJM, Griffin CM, Thompson AJ, Miller DH. The reproducibility and sensitivity of brain tissue volume measurements derived from an SPM-based segmentation methodology. J Magn Reson Imaging 15:259-267, 2002.

51. Ge Y, Grossman RI, Udupa JK, Wei L, Mannon LJ, Polansky M, Kolson DL. Brain atrophy in relapsing-remitting multiple sclerosis and secondary progressive multiple sclerosis: longitudinal data analysis. Radiology 214:665-670, 2000.

52. Udupa JK, Wei L, Samasekera S, Miki Y, van Buchem MA, Grossman RI. Multiple sclerosis lesion quantification using fuzzy connectedness principles. IEEE Trans Med Imaging 16:598-609, 1997.

53. Ashburner J, Friston K. Voxel-based morphometry-the methods. Neuroimage 11:805-821, 2000.

54. Pham DL, Prince JL. An adaptive fuzzy C-means algorithm for image segmentation in the presence of intensity inhomogeneities. Patt Recog Let 20:57-68, 1999.

55. Smith S, de Stefano N, Jenkinson M, Matthews P. Normalized accurate measurement of longitudinal brain change. J Comput Assist Tomog 25:466-475, 2001.

56. Freeborough PA, Fox NC. Modeling brain deformations in Alzheimer disease by fluid registration of serial 3D MR images. J Comput Assist Tomogr 22:838-843, 1998.

57. Freeborough PA, Fox NC. The boundary shift integral: an accurate and robust measure of cerebral volume changes from registered repeat MRI. IEEE Trans Med Imaging 16:623-629, 1997.

58. Chard DT, Griffin CM, Parker GJM, Kapoor R, Thompson AJ, Miller DH. Brain atrophy in clinically early relapsing-remitting multiple sclerosis. Brain 125:327-337, 2002.

59. Guttmann CRG, Warfield SK, Guimond A, Kikinis R, Albert MS, Jolesz FA, Weiner HL. Variability of brain atrophy estimates in multiple sclerosis (absract). Proc Int Soc Magn Reson Med 8:1188, 2000

60. Rudick RA, Fischer E, Lee J-C, Simon J, Jacobs L. Use of brain parencyhmal fraction to measure whole brain atrophy in relapsing-remitting MS. Neurology 53:1698-1704, 1999.

61. O'Riordan JI, Thompson AJ, Kingsley DPE, MacManus DG, Kendall BE, Rudge $\mathrm{P}$ et al. The prognostic value of brain MRI in clinically isolated syndromes of the central nervous system: a 10 year follow-up. Brain 121:495-503, 1998.

62. Beck RW, Trobe JD, Moke PS, Gal RL, Xing D, Batti MD et al. High and low risk profiles for the development of multiple scle- rosis within 10 years after optic neuritis: experience of the optic neuritis treatment trial. Arch Ophthalmol 121:944-949, 2003.

63. Dalton CM, Brex PA, Jenkins R, Fox NC, Miszkiel KM, Crum WR et al. Progressive ventricular enlargement in patients with clinically isolated syndromes is associated with the early development of multiple sclerosis. J Neurol Neurosurg Psychiatry 73:141-147, 2002.

64. Hickman SJ, Brex PA, Brierley CMH, Silver NC, Barker GJ, Scolding NJ et al. Detection of optic nerve atrophy following a single episode of unilateral optic neuritis by MRI using a fatsaturated short-echo fast FLAIR sequence. Neuroradiology 43: 123-128, 2001.

65. de Stefano N, Matthews PM, Filippi M, Agosta F, De Luca M, Bartolozzi ML et al. Evidence of early cortical atrophy in MS: relevance to white matter changes and disability. Neurology 60 : $1157-1162,2003$

66. Jones CK, Riddehough A, Li DKB, Zhao GJ, Paty DW. MRI cerebral atrophy in relapsing remitting MS: results of the PRISMS trial. Neurology 56(Suppl 3):A379, 2001.

67. Gasperini C, Paolillo A, Giugni E, Galgani S, Bagnato F, Mainero $\mathrm{C}$ et al. MRI brain volume changes in relapsing remitting multiple sclerosis patients treated with interferon $\beta$-1a. Mult Scler 8:119$123,2002$.

68. Losseff NA, Wang L, Lai HM, Yoo DS, Gawne-Cain ML, McDonald WI et al. Progressive cerebral atrophy in multiple sclerosis. A serial study. Brain 119:2009-2019, 1996b.

69. Paolillo A, Coles AJ, Molyneux PD, Gawne-Cain ML, MacManus D, Barker GJ et al. Quantitative MRI in patients with secondary progressive MS treated with monoclonal antibody campath 1H. Neurology 53:751-757, 1999.

70. Fisher E, Rudick RA, Simon JH, Cutter G, Baier M, Lee JC et al Eight year follow-up study of brain atrophy in patients with MS. Neurology 59:1412-1420, 2002.

71. Rovaris M, Comi G, Rocca M, Wolinsky J, Filippi M, and the European/Canadian Glatiramer Acetate Study Group. Short-term brain volume change in relapsing-remitting multiple sclerosis: effect of glatiramer acetate and implications. Brain 124:18031812,2001

72. Kalkers NF, Bergers L, Castelijns JA, van Walderveen MAA, Bot JCJ, Ader HJ, Polman CH, Barkhof F. Optimizing the association between disability and biological markers in MS. Neurology 57: 1253-1257, 2001.

73. Lin X, Tench CR, Turner B, Blumhardt LD, Constantinescu CS Spinal cord atrophy and disability in multiple sclerosis over four years: application of a reproducible automated technique in monitoring disease progression in a cohort of the interferon $\beta$-1a (Rebif) treatment trial. J Neurol Neurosurg Psychiatry 74:10901094, 2003.

74. Stevenson VL, Miller DH, Leary SM, Rovaris M, Barkhof F, Brochet B et al. One year follow up study of primary and transitional progressive multiple sclerosis. J Neurol Neurosurg Psychiatry 68:713-718, 2000.

75. Ingle GT, Stevenson VL, Miller DH, Thompson AJ. Primary progressive multiple sclerosis: a 5-year clinical and MR study. Brain 2003, in press.

76. Kalkers NF, Barkhof F, Bergers E, van Schijndel R, Polman CH. The effect of the neuroprotective agent riluzole on MRI parameters in primary progressive multiple sclerosis. Mult Scler 8:532533, 2002.

77. Coles AJ, Wing MG, Molyneux P, Paolillo A, Davie CM, Hale G et al. Monoclonal antibody treatment exposes three mechanisms underlying the clinical course of multiple sclerosis. Ann Neurol 46:296-304, 1999

78. Rice GPA for the Cladribine Clinical Study Group, Filippi M, Comi G, for the Cladribine MRI Study Group. Cladribine and progressive MS. Clinical and MRI outcomes of a multicenter controlled trial. Neurology 54:1145-1154, 2000.

79. Filippi M, Rovaris M, Iannucci G, Mennea S, Sormani MP, Comi G. Whole brain volume changes in progressive MS patients treated with cladribine. Neurology 55:1714-1718, 2000.

80. Fu L, Matthews PM, De Stefano N, Worsley KJ, Narayanan S, Francis GS et al. Imaging axonal damage of normal-appearing white matter in multiple sclerosis. Brain 121:103-113, 1998. 
81. Leary S, Davie C, Parker G, Stevenson VL, Wang LQ, Barker GJ et al. $1 \mathrm{H}$ magnetic resonance spectroscopy of normal appearing white matter in primary progressive multiple sclerosis. J Neurol 246:1023-1026, 1999.

82. Davie C, Barker G, Thompson A, Tofts P, McDonald WI, Miller DH. H magnetic resonance spectroscopy of chronic cerebral white matter lesions and normal appearing white matter in multiple sclerosis. J Neurol Neurosurg Psychiatry 63:736-742, 1997.

83. Sarchielli P, Presciutti O, Pelliciolli GP, Tarducci R, Gobbi G, Chiarini $\mathrm{P}$ et al. Absolute quantification of brain metabolites by proton magnetic spectroscopy of normal appearing white matter of patients with multiple sclerosis. Brain 122:513-522, 1999.

84. Davie C, Barker GJ, Webb S, Tofts PS, Thompson AJ, Harding AE, McDonald WI, Miller DH. Persistent functional deficit in multiple sclerosis and autosomal dominant cerebellar ataxia is associated with axon loss. Brain 118:1583-1592, 1995.

85. Pelletier D, Nelson SJ, Oh J, Antel JP, Kita M, Zamvil SS, Goodkin DE. MRI lesion volume heterogeneity in primary progressive MS in relation with axonal damage and brain atrophy. J Neurol Neurosurg Psychiatry 74:950-952, 2003.

86. Gonen O, Catalaa I, Babb JS, Ge Y, Mannon LJ, Kolson DL, Grossman RI. Total brain N-acetylaspartate: a new measure of disease load in MS. Neurology 54:15-19, 2000.

87. Filippi M, Bozzali M, Rovaris M, Gonen O, Kesavadas C, Ghezzi A et al. Evidence for widespread axonal damage at the earliest clinical stage of multiple sclerosis. Brain 126:433-437, 2003.

88. Provencher SW. Estimation of metabolite concentrations from localized in vivo proton NMR spectra. Magn Reson Med 30:672679, 1993.

89. Narayanan S, De Stefano N, Francis GS, Arnouetelis R, Caramanous Z, Collins LD et al. Axonal metabolic recovery in multiple sclerosis patients treated with interferon $\beta-1 b$. J Neurol 249:979-986, 2001.

90. Parry A, Corkill R, Blamire AM, Palace J, Narayanan S, Arnold $\mathrm{D}$ et al. $\beta$-interferon does not always slow the progression of axonal injury in multiple sclerosis. J Neurol 250:171-178, 2003.

91. Parker GJ, Wheeler-Kingshott CA, Barker GJ. Estimating distributed anatomical brain connectivity using fast marching methods and diffusion tensor imaging. IEEE Trans Med Imaging 21:505$512,2002$.

92. Ciccarelli O, Werring DJ, Barker GJ, Griffin CM, Wheeler-Kingshott CAM, Miller DH, Thompson AJ. A study of the mecha- nisms of normal-appearing white matter damage in multiple sclerosis using diffusion tensor imaging: evidence of Wallerian degeneration. J Neurol 250:287-292, 2003.

93. Barkhof F, Bruck W, de Groot JC, Bergers E, Hulsof S, Guerts J et al. Remyelinated lesions in multiple sclerosis: magnetic resonance image appearance. Arch Neurol 60:1073-1081, 2003.

94. van Walderveen MA, Kamphorst W, Scheltens P, van Waesberghe JH, Ravid R, Valk J et al. Histopathological correlate of hypointense lesions on T1-weighted spin echo MRI in multiple sclerosis. Neurology 50:1282-1288, 1998.

95. O'Riordan JI, Gawne-Cain M, Coles A, Wang L, Compston DAS, Miller DH. T1 hypointense lesion load in secondary progressive multiple sclerosis: a comparison of pre versus post contrast loads and of manual versus semi automated threshold techniques for lesion segmentation. Mult Scler 4:408-412, 1998.

96. Filippi M, Rovaris M, Rocca MA, Sormani MP, Wolinsky JS, Comi $\mathrm{G}$ et al. Glatiramer acetate reduces the proportion of new MS lesions evolving into "black holes". Neurology 57:731-733, 2001.

97. Dalton CM, Miszkiel KA, Barker GJ, Pepple TI, Panzara M, Yang $\mathrm{M}$ et al. Effect of natalizumab on conversion of gadolinium enhancing lesions to $\mathrm{T} 1$ hypointense lesions in relapsing multiple sclerosis. J Neurol 2004 (in press)

98. Brex PA, Molyneux PD, Smiddy P, Barkhof F, Filippi M, Yousry TA et al. The effect of interferon $\beta-1 \mathrm{~b}$ on the size and evolution of enhancing lesions in secondary progressive MS. Neurology 57:2185-2190, 2001

99. Filippi M, Iannucci G, Tortorella C, Minicucci L, Horsfield MA, Colombo B, Sormani MP, Comi G. Comparison of MS clinical phenotypes using conventional and magnetization transfer MRI. Neurology 52:588-594, 1999.

100. Traboulsee A, Dehmeshki J, Peters KR, Griffin CM, Brex PA, Silver $\mathrm{N}$ et al. Disability in multiple sclerosis is related to normal appearing brain tissue MTR histogram abnormalities. Mult Scler 9:566-573, 2003

101. Miller DH, Thompson AJ, Filippi M. Magnetic resonance studies of abnormalities in the normal appearing white matter and grey matter in multiple sclerosis. J Neurol 250:1403-1419, 2003.

102. Grimaud J, Millar J, Thorpe JW, Moseley IF, McDonald WI, Miller DH. Signal intensity on MRI of basal ganglia in multiple sclerosis. J Neurol Neurosurg Psychiatry 59:306-308, 1995. 